\title{
Palmprint Recognition System Robust to Occlusion using Gabor with 2D-2DPCA
}

\author{
Sunmeet Kaur \\ M. Tech Student \\ Gyan Ganga Institute of Technology \& Sciences \\ Jabalpur (M.P)
}

\author{
Preeti Rai \\ Associate Professor \\ Gyan Ganga Institute of Technology \& Sciences \\ Jabalpur (M.P)
}

\begin{abstract}
Biometric based identification is one of the fields emerging recently, used as a form of identification and access control in various places to ensure security. Palmprint is one of the most unique and stable biometric characteristics. A palmprint contains different elements, including essential lines, wrinkles, edges, particulars focuses, solitary focuses and surface. It is essential to extract only useful segment of the palmprint image in the form of Region of Interest (ROI), which has highest concentration of potential features. It helps the system by reducing the size of template, speeding up the process and adds accuracy. Thus, palmprint recognition system's performance can be improved when size of the palm images can be reduced first and then features are extracted from the images to recognize the identity of an individual. This paper presents a texture based palm print recognition method which employ 2D Gabor filter to extract texture information from the central part of hand and use Twodirectional Two-dimensional Principal component analysis (2D-2DPCA) methods for dimension reduction. The test and training images are compared in terms of calculating Euclidean distance between them using KNN classifier. The proposed system is also robust to occlusion and can verify the user by comparing features of from nonoccluded region. All tests are performed on 100 classes of the Hong Kong PolyU palmprint database. The Hong Kong PolyU database comprises of 7960 pictures captured from 199 people, 398 palms. It comprises of 20 pictures of each palm and it is the biggest palmprint database freely accessible.
\end{abstract}

\section{General Terms}

Palmprint recognition, feature extraction, security et. al.

\author{
Keywords \\ Pattern Recognition, Security, Palm Print, Biometrics
}

\section{INTRODUCTION}

Recent years have seen significant increased in biometrics recognition applications, partly due to recent advances in technology, partly due to increase demands for security and also due to human cost saving reasons. Biometrics is able to provide for confidential financial transactions and personal data privacy. Venture wide system security frameworks, government IDs, secure electronic management of an account, contributing and other budgetary exchanges, retail deals, law requirement, and wellbeing and social administrations are as of now profiting by these advances. Biometric using person identity traits is fast, easy-to-use, defined, reliable and cost-effective over outdated knowledge based and tokenbased methods. A biometric framework contains fundamentally a picture capturing module, a feature extraction module and a pattern matching module as appeared in Figure 1. An image capturing module acquires the raw biometric data of an individual by means of a sensor. After which feature extraction module progresses the quality of the captured image. Whereas database module stores the biometric template information of enrolled Persons. Pattern matching module compares the extracted features with the stored templates, which in-turn generates match score. The biometric systems offer numerous benefits over outdated authentication systems. The problem of information security gives the protection of information ensuring only authorize users are able to access the information. They are required the person being authenticated to be present at the point of authentication. Thus biometric-based authentication method is most secure system [1].



Figure 1: Biometrics System Module 
Palmprint comprise of Principal lines, texture, ridges, minutiae points, singular points, and wrinkles are viewed as valuable elements for palmprint representation. Various features can be extracted from palmprint at diff erent image resolutions that can be used to match to identify any person. For components such as minutiae points, ridges, and singular points, a high-resolution image, as shown in the Figure 2 (a), with at least 400 dpi (dots per inch) is required for feature extraction. However, features like principal lines and wrinkles can be obtained from a lowresolution palmprint image, as shown in the Figure 2 (b), with less than $100 \mathrm{dpi}$. All in all, high resolution images are basic for a few applications such as law enforcement, where ridges, singular points, and minutiae points are extracted and matched in latent prints for identification and verification purposes [2].

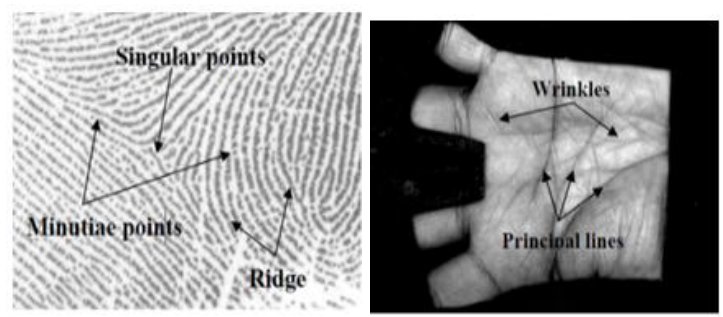

Figure 2: Palmprint Features: (a) High resolution (b) Low resolution

Palmprint biometrics offers the several advantages. Palmprint has large surface area as compare to fingerprint, so it has more features. It is very difficult to identify twins by the face and iris biometrics. Palmprint of two identical twins are diff erent so they can be easily identified [3]. An individual is less likely to damage a palm than a fingerprint. Low resolution scanners can be used for capturing palmprints. Palmprint biometrics has merits such as permanence, robustness, high acceptability, high accuracy, and cost-eff ectiveness. Palmprint recognition is promising biometrics feature for use in following applications such as physical access control, time and attendance, logical access control, forensic applications, transactional biometrics etc.

Restricted work has been accounted for on palmprint based recognition notwithstanding of its significant highlights. Endeavors have been made to manufacture a palmprint based recognition system based on structural features of palmprint like principle lines [4], local binary pattern histograms [5]. There likewise exists frameworks based on statistical features of palmprint extracted using Histogram Fourier transforms [5], Discrete Cosine Transforms [6,7], Wavelet transforms[9], principal component analysis(PCA) [10], Gabor filter [11], Neural networks [12], statistical signature [13], hand geometry [14] and Radon transforms [24]. Furthermore, there are multi-modal biometric systems that use an approach called fusion. Fusion is a promising approach that may increase the accuracy of systems so that chance of spoofing can be decreased. Fusion means combining other biometric traits such as face, fingerprints, palm veins and iris with palm prints [15].

In recent years palmprint images are captures using CCD scanners and digital camera. CCD based scanner gives the high quality of the palmprint image [16] because the images are captured in a controlled environment. A CCDbased palmprint scanner requires an appropriate choice of focal point, camera, and light sources. Simple scanner or camera are also used by the some of the researchers [17]. Accumulation approaches in view of advanced scanners, computerized cameras and camcorders require less eff ort for framework design and can be found in office environments the issue with this framework are that client may neglect to give the example in the event that he is harmed or physically tested. Hence there is a need to build a system which is

- Constraint free image acquisition: Device to acquire hand image should be constraint free so that physically challenged or injured people can provide biometric sample

- Robust to interpretation of hand picture on the scanner: System must have the capacity to extricate palmprint autonomous of translation, rotation or placement of hand on scanner surface.

- Robust to occlusion of hand image: If customer uncovered halfway palmprint because of wounds or physically tested, framework ought to have the capacity to check that individual effectively.

- High Accuracy: High Accuracy with reasonable price to suit high end security applications.

This paper proposes a palmprint based biometric framework that tends to a portion of the above attributes.

\section{LITERATURE REVIEW}

In the last few years, Computer based personal identification is being implemented using various biometric techniques. In these biometric techniques palm print is also an important technique, where lines and points are extracted from palms for personal identification. The palm has various unique features for identification. From last few decades, different researchers describe different approaches for personal identification of users using palm print. Ahmad Harb et al. [8] used three different methods for extraction of palm line, the first one was Gabor filter which is used to obtain the palm texture and then to extract the principal lines. The second method they used was canny filter as an edge detector and the third one is inspired from Radon Transform property called Modified Finite Radon Transform. M. O. Rotinwa-Akinbile et al. [4] used extracted k-points DFT coefficients as discriminating features for recognition and identification purposes using correlation technique, power spectral matching and Euclidean distance measure. Vikas varshney et al. [7] used hybrid Discrete Wavelet Transform - Discrete Cosine (DWT-DCT) technique for feature extraction. Kasturika B. Ray and Rachita Misra [18] proposed a palm print recognition system by using Hough Transform for feature extraction. Hough Transform (HT) is one of the most powerful tools for finding graphical elements in an image. Gaurav Jaswal et al. [10] proposed a palm print recognition system based on a combination of 2D- DWT and Kernel PCA for palm print based biometric recognition, On the basis of these features, KNN based three distance measures are used separately to find the closest match between the images. Hafiz Imtiaz and Shaikh Anowarul [19] Fattah proposed a system for palm print recognition based on spatial feature extraction. The entire image is segmented into several narrow-width bands and the task of feature extraction is carried out in each band by using two dimensional Fourier transforms. A principal component based analysis is performed to further 
reduce the dimensionality of the feature space, which results in lower computational complexity. Finally, recognition task is carried out using a distance based classifier. Gupta et al. [15] proposes a multimodal biometric system using face modality combined with palm print and palm vein modality. The proposed methodology applies Local Statistical method where pre-defined block of DCT coefficient is employed to calculate standard deviation and store them as feature vector. Matching is done using distance between feature vector of testing and training data set. Deepti Tamrakar and Pritee Khanna [9] proposed a palmprint recognition by combining different texture extraction approaches with high accuracy. The Region of Interest (ROI) of palmprint image is first decomposed into different frequency time sub-bands by wavelet transform up-to two levels and only the approximate image of two levels is selected, which is known as Approximate Image ROI (AIROI). This AIROI carries the information of principal lines of the palm. The Competitive Index is used as the features of the palmprint, in which six Gabor filters of different orientations convolve with the palmprint image to extract the orientation information from the image. Dominant features are selected using winner-take-all strategy for each pixel, which is known as Competitive Index. After which, PCA is applied to select highly uncorrelated Competitive Index features, to reduce the dimensions of the feature vector, and to project the features on Eigen space. Euclidean distance metrics is used to find the similarity of two palmprints. Deepti Tamrakar and Pritee Khanna [20] has proposed Competitive coding method for feature extraction and then KNN Classifier is used for palmprint verification. Deepti Tamrakar and Pritee Khanna [21] presents a palmprint recognition approach based on the local distribution of uniform patterns where, ULBP histograms are considered as features and are able to handle occlusion up to $36 \%$. Badrinath G. S. [22] proposes a palmprint based verification system using low-order Zernike moments of palmprint sub-images. The Zernike moments of each and every sub-image of palmprints that are enrolled or captured live matched using Euclidean distance for verification. Deepti Tamrakar and Pritee Khanna [23] in this paper used Gabor filters of six different orientation to extract the orientation information from the image. After this Competitive index scheme is applied in the approximate band of DWT. Principal component analysis (PCA) is used to reduce the size of the feature vector and project the features in eigen space. Two different palmprints are matched using Euclidean distance metrics. Deepti Tamrakar and Pritee Khanna [24] proposed a palmprint recognition system based on RDF descriptor which is the combination of Radon, Dual tree complex wavelet, and Fourier transforms, their combined properties help to explore efficiency and robustness of RDF descriptor for palmprint identification.

\section{PROPOSED PALMPRINT RECOGNITION SYSTEM}

The Researched had mainly worked on non-occluded palm image. The Researcher have rarely discussed the following challenges that can be faced in palm print recognition are as follows: -

1. Ladies/Housewives: The ladies involved in cooking get frequent cuts in their hands during cutting vegetables which changes the palms beyond acceptance range
2. Tobacco Chewers: Most of the population involves in biting tobacco (khaini) which must be granulated well on the palm with fingers. The pounding of tobacco is finished with lime (chuna). With time, the procedure dispenses with all the palm lines of the client. Such clients can't be even enlisted to the biometric framework.

3. Change in Skin: It has been seen in many people that their skin changes with time. The upper layer of the skin comes out on a regular time period during which the system rejects them due to change in pattern. With time as the new skin gets harder, they may get acknowledged by the framework once more. In case there is a generally high rate of progress in the examples with the new skin, the dismissal holds on.

4. Farmers and Factory Workers: With the passage of time, the farmers and the factory workers lose their palm pattern due to constant tight holding of the farm and industry equipment and friction of it.

5. Shrinkage in extreme cold: The dismissal rate increments in winters as the skin gets contract in extraordinary chilly in the northern districts of India.

6. Women applying Mehendi: The ladies apply Mehendi during various traditional functions or otherwise and this leads to the rejection of their palm prints.

So, in our work we have proposed a palmprint recognition system that will work with normal palmprints i.e. palmprints without occlusion as well as palmprints with occlusion. The proposed palmprint recognition system instigates with the successful extraction of ROI containing the most relevant information of palm from the palm image. To make the system occlusion free, it is necessary to extract local features from palm. Local information of the palm carries the most discriminative features to recognize an individual, and is gathered by dividing the palm image into $\mathrm{m} \times \mathrm{n}$ number of sub-images. Bi-level wavelet decomposition applied on a sub-image gives Approximation Palm Sub-Image (APSI) corresponding to it. APSIs contain potential information of ROI image. APSIs thus obtained might suffer from illumination and several orientation variations. A set of Gabor filters with six orientations is then applied to get the real Gabor space which contains vital information of the palm and is less sensitive to noise and illumination variations. The real Gabor space is a quite high dimensional space. It is further projected to horizontal and vertical directions of $2 \mathrm{D}$ 2DPCA subspace for dimension reduction and selection of the most discriminated feature subset. The block diagram of the proposed system is given in Figure 3. For each palm image, feature vectors of all APSIs corresponding to this image are concatenated in a row, and form features of the palm image as given in Equation (1), where Fi represents features of ith image.

$F_{i}=\left[F V_{1,1}^{i}, F V_{1,2}^{i}, \ldots F V_{m, n}^{i}\right]$

This palmprint recognition system must not only be able to recognize a person from non-occluded normal palm images, but also be invariant to occluded palm images. These occluded palm images are not present in the training set of images, but are present in the images given for testing. Before showing that the system is working well for the occluded palm images, its performance is checked for non-occluded palm images. Later the performance is tested on occluded palm images. Each module of the system is explained in following subsection. 


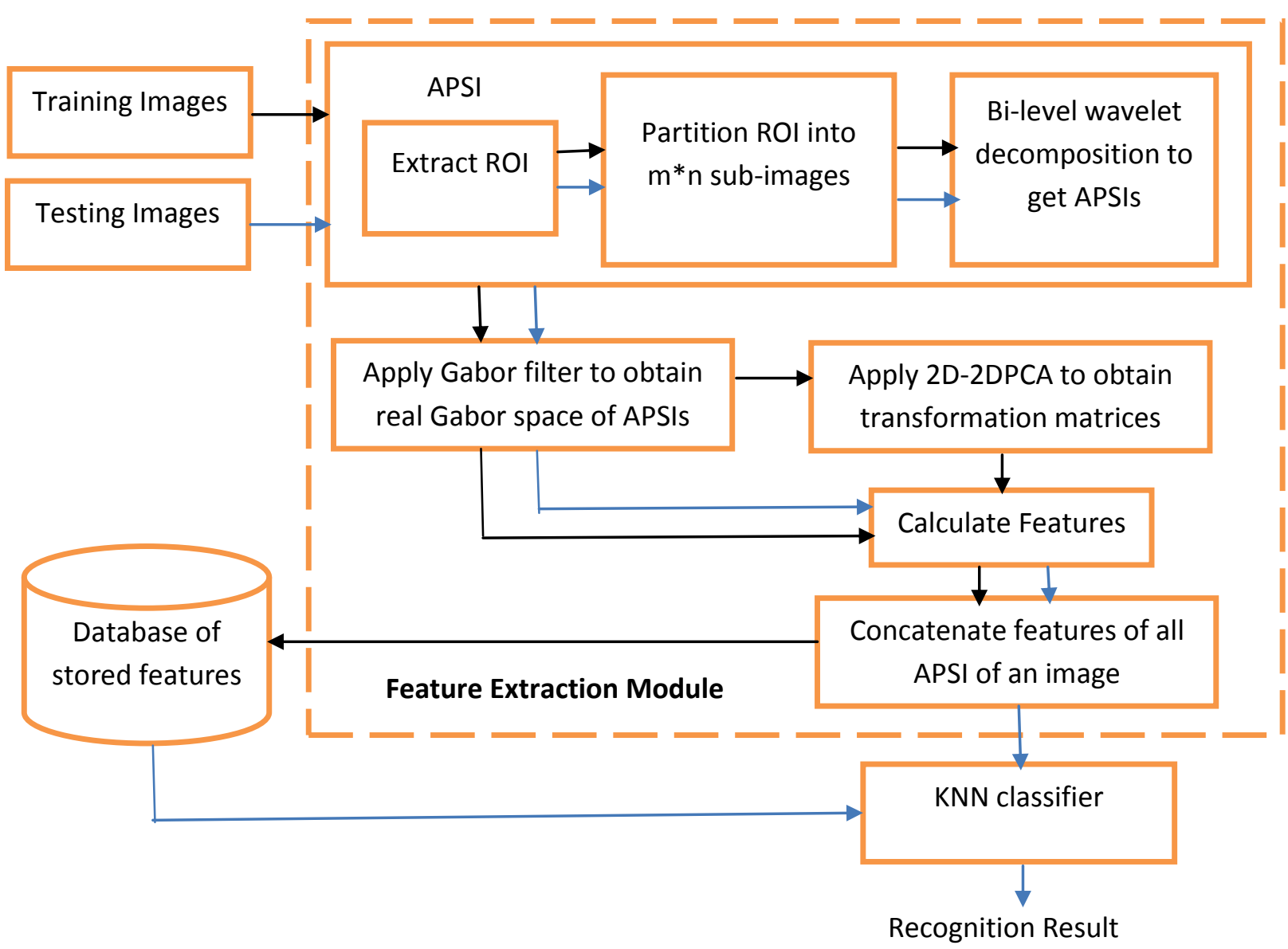

Figure 3: Block Diagram of the Proposed Recognition System

\subsection{Image Acquisition}

The palmprint images are taken from Hong Kong PolyU palmprint database which consists of 7960 images captured from 199 individuals, 398 palms. It consists of 20 images of each palm and it is the largest palmprint database publicly available.

\subsection{Preprocessing}

Palmprint picture ought to be adjusted to a predefined general arrangement to encourage predictable ROI extraction, Before the feature extraction the following steps are used to align palmprint images:

- Crop the palmprint images.

- A square of $128 \times 128$ pixels is extracted that is known as the ROI.

- Then palmprint images are converted to grayscale images as shown in Figure 4.



Original image Cropped image Gray Image

Figure 4: Pre-processing Steps

\subsection{Feature Extraction}

The main purpose to extract features from a palmprint image is to get particular information about the orientation of palmprint lines. It usually done by using group of filters with different orientations. In this proposed work, two well-known techniques for feature extraction are combined to extract the feature of the palmprint image. ROI obtained after pre-processing of palmprint image is decomposed up to two levels by using discrete wavelet transform for different wavelet filter. Gabor filter with six different orientations convolves with approximate coefficients of ROI. Two-directional Two-dimensional Principal component analysis (2D-2DPCA) has been used to reduce the number of features. The feature extraction technique used in this work referred as Gabor $+2 \mathrm{D}$ 2DPCA that uses Gabor filter on two directional twodimensional PCA and subspace projection method TwoDirectional Two-dimensional PCA on Approximation palm image (APSI) obtained by using Bi-level wavelet decomposition, referred as APSI + 2D-2DPCA. The steps for calculating features are as follows:

1. To extract local features of palm, ROI obtained from the palm image is divided into $m \times n$ sub-images as shown in Figure 5.

2. Bi-level wavelet decomposition applied on a subimage gives Approximation Palm Sub-Image (APSI) corresponding to it. APSIs contain potential information of ROI image.

3. A set of Gabor filters with six orientations is then applied to get the real Gabor space which contains vital information of the palm and is less sensitive to noise and illumination variations. The real Gabor space is a quite high dimensional space. 
4. It is further projected to horizontal and vertical directions of 2DPCA, i.e., 2D-2DPCA subspace for dimension reduction and selection of the most discriminated feature subset. The feature extraction module works through these consecutive steps discussed above, which are visualized in Figure 6.

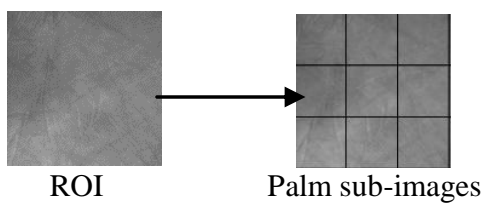

After feature extraction Entropy of each APSI is used to identify non-occluded sub-images in the test ROI. Matching is performed between corresponding features of non-occluded APSI identified in test palmprint and enrolled palmprints by using Euclidean distance and $\mathrm{K}$ nearest neighbor $(\mathrm{KNN})$ classifier for identification. The details of each of the technique used in feature extraction are given in the following subsections.

Figure 5: Obtaining sub-images from ROI

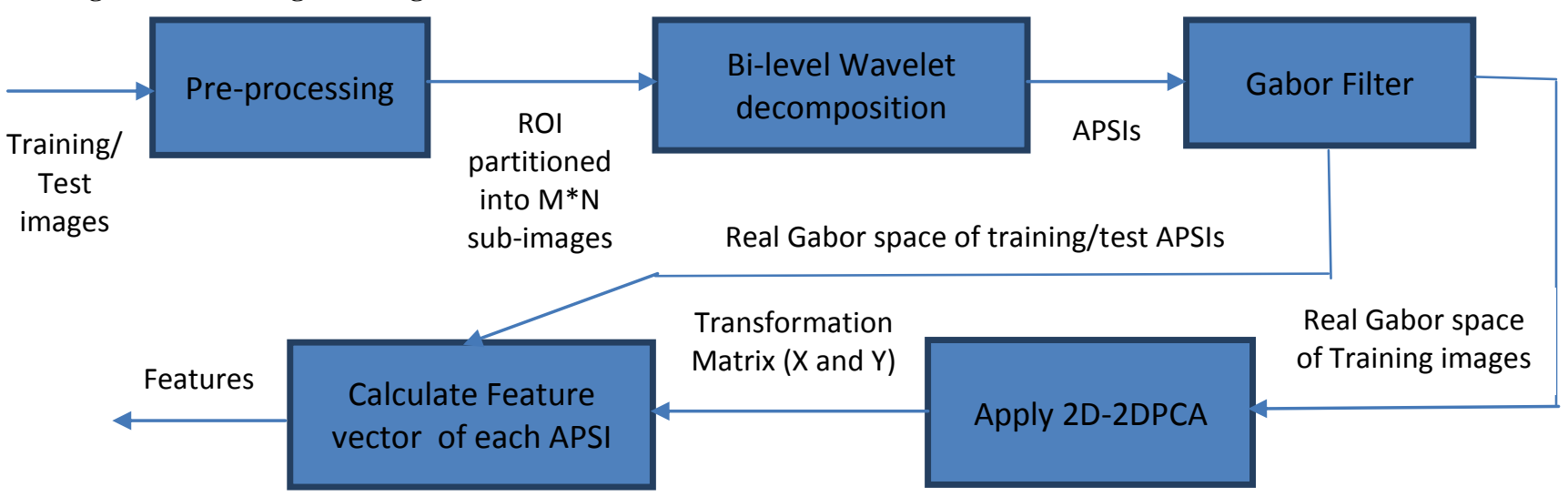

Figure 6: Feature extraction with Gabor based 2D-2DPCA on APSI

\subsubsection{Discrete Wavelet Transform}

The wavelet transforms as compared to the traditional Fourier analysis, has better space-frequency localization. Wavelet decomposition is time frequency signal analysis method. Thus, it is suited for analyzing images where most of the informative content is represented by components localized in space, such as, edges and borders and by information at different scales or resolutions, with large and small features. Different frequencies, including approximate coefficient and detailed coefficient (horizontal, vertical and diagonal) can be acquired by Wavelet Decomposition.

APSI is generated by applying bi-level wavelet decomposition on the cropped palm image i.e. ROI. Here, DWT is used to decompose the extracted ROI into lower resolution representation. Instead of taking the original image, the approximation coefficients of second level decomposition of ROI are selected for feature extraction to increase the accuracy and speed of the system. Detail coefficients give good time resolution and poor frequency resolution; while approximation coefficients give good frequency resolution and poor time resolution. The energy compaction is more into the approximation coefficients as compared to detail coefficients. High frequency components give the sharp edges in the detail image. In the approximation sub-band, palm image does not loose the basic appearance of the palm. At each level of decomposition, wavelet transform is computed by successive low-pass and high-pass filtering of ROI. Highpass filter produces detailed information; whereas lowpass filter associated with scaling function results in coarse approximations of ROI. At each level, the image of size $\mathrm{M} \times \mathrm{M}$ is decomposed into four sub-bands (approximation, horizontal, vertical, and diagonal) of size $\mathrm{M} / 2 \times \mathrm{M} / 2$ with diff erent spatial resolutions, frequency characteristics, and directional.
Among all four coefficients, approximation coefficients contain low frequency components of ROI and referred as APSI (approximation palm sub-image) in this work. APSI carries whole information of the ROI and hence, considered for further processing. Here, wavelet decomposition aids in dimension reduction of palm images also while maintaining potential palm information. Using APSI, instead of ROI, for feature extraction results in a reduced sized feature vector which may lead to a faster palmprint recognition.

\subsubsection{Gabor Filters}

Gabor filter named after Dennis Gabor is a linear filter, which is used for analyzing the specific frequency content in an image in specific directions. Gabor filter is a frequency and orientation selective filter. Gabor filter is widely used in area of image processing, pattern recognition applications and computer vision. Gabor filters are precise in time frequency localization. Two dimensional Gabor filters were proposed in 1980 by Daugman as a framework for understanding orientation selective and spatial domains. In spatial domain, a 2D Gabor filter, $h(x, y)$ is a Gaussian kernel function modulated by sinusoidal plane of particular frequency and orientation.

$h(x, y)=s(x, y) * g(x, y)$

Where, $s(x, y)$ is a sinusoidal plane of particular frequency and orientation $g(x, y)$ is a 2D Gaussian function, and both parameters are defined as:

$s(x, y)=\exp \left(2 \pi j u x^{\prime} \omega+\emptyset\right)$

$g(x, y)=\frac{1}{2 \pi \sigma^{2}} \exp \left(-\frac{\left(x^{2}-y^{2}\right)}{\sigma^{2}}\right)$

Hence Gabor filter equation is: 
$h(x, y, \sigma, \theta, u)=\frac{1}{2 \pi \sigma^{2}} \exp \left(-\frac{\left(x^{2}+y^{2}\right)}{\sigma^{2}}\right) \exp \left(2 \pi j u x^{\prime} \omega+\emptyset\right)$

Where, $x^{\prime}=(x \cos \theta+\sin \theta)$ and $j=\sqrt{-1}$

Gabor function parameters specified by frequency $(u)$, an orientation $(\theta)$ of sinusoid, spatial variables $(x, y)$, the offset phase $(\varnothing)$ of sinusoidal function, standard deviation $(\sigma)$, spatial aspect ratio $(\omega)$. These parameters affect accuracy of proposed work as well as size of feature vectors.

With $\omega=1$ and $\emptyset=0$, the equation (3) become as:

$h(x, y, \sigma, \theta, u)=\frac{1}{2 \pi \sigma^{2}} \exp \left(-\frac{\left(x^{2}+y^{2}\right)}{\sigma^{2}}\right) \exp \left(2 \pi j u x^{\prime}\right)$

(4)

The filter has real and imaginary parts for representing orthogonal directions. From above equation (4), the real and imaginary part is as follow, respectively:

$$
R h(x, y, \sigma, \theta, u)=\frac{1}{2 \pi \sigma^{2}} \exp \left(-\frac{\left(x^{2}+y^{2}\right)}{\sigma^{2}}\right) \cos \left(2 \pi j u x^{\prime}\right)
$$

$$
\operatorname{Ih}(x, y, \sigma, \theta, u)=\frac{1}{2 \pi \sigma^{2}} \exp \left(-\frac{\left(x^{2}+y^{2}\right)}{\sigma^{2}}\right) \sin \left(2 \pi j u x^{\prime}\right)
$$

(6)

Gabor function has cos and sine functions of sinusoidal plane and is symmetric to orientation. The cos function is the real part of Gabor filter that follows the properties of even function, while the sine function is the imaginary part of the Gabor filter and follows the properties of the odd function. Real and imaginary part of the Gabor filters are used for analysis of textures. Gabor filter is applied for finding the line information of the palmprint image in different orientations. The parameters of the Gabor filter play important roles for finding the information of the textures. The success of the developed algorithm highly depends on the Gabor filter parameters; namely $\sigma$, u and size. Value of standard derivation $(\sigma)$ of Gaussian directly effects on blurring of the image. The sharpness of the features of the palmprint image depends on the frequency of the sinusoidal plane. The orientation of the extracted line features of the palmprint image depends on the orientation of the Gabor filter. The selection of these parameters affects not only the accuracy of the developed system, but also the size of templates to be stored in the database. Furthermore, the size of the selected Gabor filter directly affects the speed of the system. If the parameters of the Gabor filter are determined, then Gabor filter coefficients can be easily calculated. The optimal parameters of Gabor filter for palmprint recognition, which are used in this work, are shown in the Table 1.

Table 1: Optimal Parameters of the Gabor filter

\begin{tabular}{|c|c|c|c|}
\hline Size & $U$ & $\sigma$ & $\theta$ \\
\hline $9 * 9$ & 0.0565 & 0.02 & $45^{\circ}$ \\
\hline
\end{tabular}

\subsubsection{D-2D Principal Component Analysis}

Principal components are uncorrelated variables which are converted from a set of observations of possibly correlated variables. The number of principal components is less than or equal to the number of original variables [23]. This transformation is defined in such a way that the first principal component has highest variance. PCA is used to reduce feature dimensions but there is a problem in PCAbased palm representation and recognition approach is that the 2D palm image matrices must be previously converted into 1D image vectors row by row or column by column, this increase computational cost.
To overcome these problems, a method was proposed named 2-dimensional principal component analysis (2DPCA), this method directly computes eigenvectors from original image matrix, so this is also named image covariance matrix without matrix-to-vector conversion. We can also say, image matrices can be directly processed and there is no need to transform images matrices into Column or row vectors, because size of image covariance matrix is equal to or less then with the size of original images, and the size obtained in 2DPCA of image covariance matrix is very small compared with the size of a covariance matrix in PCA. As 2DPCA works in row direction, it reduces dimensions in row direction and a procedure named alternate 2DPCA is works in column direction, it reduces dimensions in column direction. As by using 2DPCA optimal projection is given in row direction because it works in row direction only, similarly, by using alternate 2DPCA the optimal projection is given in column direction. However, the chief drawback of 2DPCA and alternate 2DPCA is that they both required too much coefficients for image representation. Solution of this problem can be given if there is a procedure which project image feature matrix both in column and row directions simultaneously. So we have proposed a method named 2D2DPCA (two-directional two-dimensional principle component analysis). 2D-2DPCA is a simultaneous way of presenting 2DPCA and alternate 2DPCA, it's workflow is shown in Figure 7.



Figure 7: Work flow of two directional 2DPCA

\subsection{Use of Entropy to identify occluded Palm}

In this work, the concept of entropy is used to identify occluded APSIs. Entropy is a statistical measure of randomness that can be used to characterize the occluded part in images. The randomness in the occluded part is low as there is less texture information as compared to the nonoccluded part. Thus, the entropy of the occluded part is always less than the non-occluded part.

During testing, the occluded part in the palm region is detected on the basis of entropy of the test image and a predetermined threshold. The threshold is calculated during the training phase of the system. While training, entropy $(E m, n i)$ of the real Gabor space for $(m, n)$ th block of each ith image, and average entropy $(\mathrm{AEm}, \mathrm{n})$ of real Gabor space of each $(m, n)$ th APSI of all training images are calculated as

$$
E_{m, n}^{i}=-\sum_{B} P_{m, n}^{B} \log _{2} P_{m, n}^{B}
$$


where, B represents gray levels and $P_{m, n}^{B}$ is the probability of $(\mathrm{m}, \mathrm{n})$ th block having Bth gray level.

$$
A E_{m, n}=\frac{1}{I} \sum_{i=1}^{I} E_{m, n}^{i}
$$

where, $I$ is the number of training images.

This average entropy of the real Gabor space of each APSI is treated as the threshold to determine the occluded or non-occluded parts in the test image during testing phase. During testing phase, the entropy of the real Gabor space for each APSI in the test image is calculated in the similar way. To find occluded APSI in the test image, entropy of each test APSI, say (TEm,n), is compared with the average entropy, (AEm,n), corresponding to this APSI. If TEm, $n$ exceeds half of the AEm,n, the APSI is considered intact (non-occluded) and hence its features are utilized for classification. Otherwise, APSI is considered as occluded. Features obtained from occluded APSI would not be used further for classification.

\subsection{KNN-Classifier with Euclidean Distance Metrics}

$\mathrm{K}$-nearest neighbor is a supervised learning calculation where the after effect of new case of palmprint is grouped on the premise of dominant part of K-nearest neighbor class. To find out closest neighbor, calculate Euclidean distance to each training sample and then sort it in ascending order and then find $\mathrm{K}$ nearest training samples. The motivation behind this algorithm is to classify a query palmprint image based on attributes and training samples. Given a palmprint image, we find $\mathrm{K}$ number of training palmprints closest to the query palm. The classification is majority vote among the classification of the $\mathrm{K}$ objects. $\mathrm{KNN}$ classifier can be breaking any tie at random. KNN classifier is Robust to noisy training data and effective also if training data is large.

Verification of a query palmprint image is determined by the class of its k-nearest neighbors. If class of a query palmprint image is same as output of its K-nearest neighbor classifier, then palmprint is matched with training samples otherwise not matched. In order to define classes of K-nearest neighbors on the basis of distance we have used Euclidean distance formula in our work. The formula for Euclidean Distance is as follows:

$d(p, q)=\sqrt{\sum_{i-1}^{N}(q i-p i)^{2}}$

\section{EXPERIMENTAL SETUP AND RESULTS}

Tool Used: All simulations are performed using MATLAB. MATLAB stands for matrix laboratory. MATLAB developed by Math Work. It is fourthgeneration high-level programming language. It easily works on matrix problems. Plotting of function or data, implement algorithm is easy with the help of MATLAB. In MATLAB, there is various inbuilt function. There is a toolbox in MATLAB which contain various matrix operations. Basic structure of MATLAB is matrix and the data type is an Array. The robustness of the proposed approach is tested on normal and artificially occluded palmprints. i) Testing on normal palmprints: In the first set of experiments, the proposed method is tested on normal palmprints as shown in Figure 8. Table 2 illustrates the correct recognition rate (CRR) of the proposed feature extraction approaches on database containing normal palm image with different Daubechies filter i.e. Db1, Db2 and Db3. The Db1 filter achieves highest recognition rate; which is 98.6014 for PolyU database.


Figure 8: Normal Palmprints

Table 2: CRR obtained for normal palmprints with proposed method

\begin{tabular}{|c|c|}
\hline Wavelet filter & CRR \\
\hline \hline Db1 & 98.6014 \\
\hline \hline Db2 & 91.5825 \\
\hline \hline Db3 & 89.5623 \\
\hline
\end{tabular}

ii) Testing on artificially occluded palmprints: In real life, the size and position of occlusion in palmprints vary for different reasons. Some examples of palmprints having natural occlusion due to injury and tattoo are shown in Figure 9. As the database of naturally occluded palmprints is not yet available publicly, the proposed method is validated on artificially occluded test ROIs as shown in Figure 10 and Table 3 illustrates the correct recognition rate(CRR) of the proposed system for artificially occluded images with occlusion up to 30 percent introduced at any portion on it.
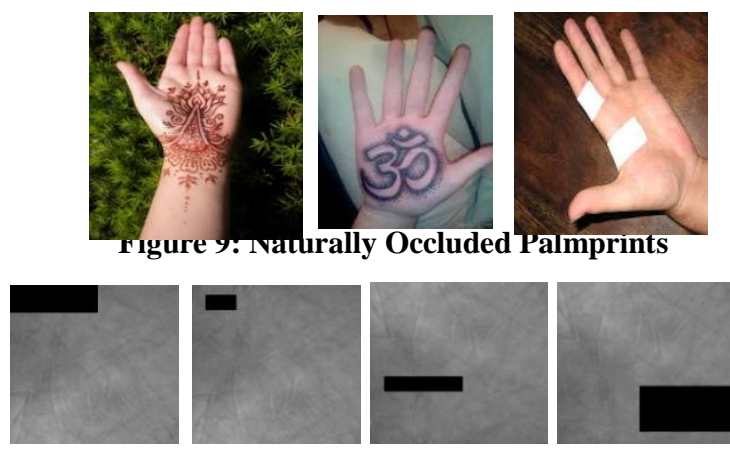

Figure 10: Artificially Occluded Palmprints

Table 3: CRR obtained for artificially occluded palmprints with proposed method

\begin{tabular}{|c|c|c|}
\hline Wavelet Filter & Occlusion \%age & CRR \\
\hline \hline Db1 & 2 & 97.9021 \\
\hline \hline Db1 & 5 & 95.8042 \\
\hline \hline Db1 & 10 & 95.4545 \\
\hline \hline Db1 & 15 & 94.4664 \\
\hline \hline Db1 & 20 & 100 \\
\hline \hline Db1 & 22 & 99.3007 \\
\hline \hline Db1 & 25 & 98.5337 \\
\hline Db1 & 30 & 98.5337 \\
\hline
\end{tabular}




\section{CONCLUSION}

In this paper, we presented a palmprint recognition system by using wavelet transform, Gabor filter, together with $2 \mathrm{D}$ 2DPCA for feature reduction. Wavelet transform contributed largely to this research as it does not only help in reducing the image size, but also retains the important palmprint information that is vital to the recognition. Thus, it is suited for analyzing images where most of the informative content is represented by components localized in space, such as, edges and borders and by information at different scales or resolutions, with large and small features. Only $\mathrm{Db}$ wavelet families have property of symmetric and regularity. In this study $\mathrm{db} 1$ wavelet filter gives higher CRR and the proposed system works well with non-occluded as well as occluded palm images. In future, it is required to explore easy and valid method for segmentation of ROI of palmprint image that is taken by digital camera. We can also use fusion of two different distance classifier such as Euclidean distance, angular distance and hamming distances to improve the result.

\section{REFERENCES}

[1] Regin Joy Conejar, JunWoo Jo, Jaeeon Bae, HaengKon Kim, "A Study of Different Biometrics Recognition Technology and Its Application", BSBT, ISBN: 978-1-4673-9843-5/15, pp:12-15, November 2015.

[2] K. Suhasini, S. Sanjushree and C. Palanisamy, "Efficiency of Various Approaches for Feature Extraction in Palm Recognition", IJCA, ISSN (Online): 0975 - 8887, pp. 21-24, October 2014.

[3] Kong A., Zhang D., Lu G., "A Study of Identical Twins Palmprint for Personal Verification", Pattern Recognition, Vol. 39, pp. 2149-2156, 2006.

[4] M. O. Rotinwa-Akinbile A.M. Aibinu1 and M. J. E. Salami, "Palmprint Recognition Using Principal Lines Characterization", UNPAR, pp. 278-282, December 2011.

[5] Wafa El-Tarhouni1, Larbi Boubchir2, Noor AlMaadeed3, Mosa Elbendak1 and Ahmed Bouridane1, "Multispectral Palmprint Recognition Based on Local Binary Pattern Histogram Fourier Features and Gabor Filter", publisher: IEEE, October 2016.

[6] Hafiz Imtiaz, Shubhra Aich and Shaikh Anowarul Fattah, "Palm-print Recognition Based on DCT Domain Statistical Features Extracted from Enhanced Image", ICEEICT, April 2014.

[7] Vikas Varshney, Rashmi Gupta and Prerna Singh, "Hybrid DWT-DCT based Method for Palm-print Recognition”, ISSPIT, pp. 000007-000012, December 2014.

[8] Ahmad Harb, Mahmoud Abbas Ali Cherry, Hussein Jaber and Mohamad Ayache, "Palm Print Recognition”, ICABME, pp. 13-16, September 2015.

[9] Deepti Tamrakar, Pritee Khanna, "Palmprint Recognition by Wavelet Transform with Competitive Index and PCA", Publisher: IEEE, pp: 1581- 1585, September 2011.
[10] Gaurav Jaswal, Ravinder Nath and Amit Kaul, "Multiple Resolution based Palmprint Recognition using 2D-DWT and Kernel PCA", ICSC, pp. 210215, March 2015.

[11] Gaurav Jaswal, Ravinder Nath, Amit Kaul,” Texture based Palm Print Recognition using 2-D Gabor Filter and Sub Space Approaches", ISPCC, pp: 344- 349, Sept. 2015.

[12] Prasetya Aria Wibawa Tjokorda Agung B W and Febryanti Sthevanie, "Palm Print Recognition Using Competitive Hand Valley Detection, Local Binary Pattern and Probabilistic Neural Network", ICITSI, pp:" 105-110

November 2014

[13] Naruemol Chumuang, Mahasak Ketcham, "Intelligent Handwriting Thai Signature Recognition System based on Artificial Neuron Network", Publisher: IEEE, January 2015.

[14] Odgerel Ayurzana, Bumduuren Pumbuurei, Hiesik Kim, "A Study of Hand-Geometry Recognition System”, Publisher: IEEE, October 2013.

[15] Mr. Aditya Gupta, Mr. Abhijit Malage, Mr. Dhiraj More, Miss Priya Hemme, Miss Prayanti Bhautmage and Miss Duhita Dhandekar, "Feature Level Fusion of face, palm Vein and palm print Modalities using Discrete Cosine Transform", ICAETR (online), ISSN: 2347-9337, August 2014.

[16] Zhang D., Kamel M., "Survey of Palmprint Recognition, Pattern Recognition", Vol. 42, pp.1408 1418, (2009).

[17] Aishwarya D, Gowri M and Saranya R K, "Palm Print Recognition Using Liveness Detection Technique", ICONSTEM, pp. 109-114, December 2016.

[18] Kasturika B. Ray and Rachita Misra Department of IT, "Palm Print Recognition using Hough Transforms", ICRCICN, pp. 422-425, December 2015.

[19] Hafiz Imtiaz and Shaikh Anowarul Fattah, "A Spectral Domain Feature Extraction Scheme for Palm-print Recognition”, WCSP, October 2010.

[20] Deepti Tamrakar and Pritee Khanna," Analysis of Palmprint Verification using Wavelet Filter and Competitive Code", publisher: IEEE, CICN, pp:2025, November 2010.

[21] Deepti Tamrakar and Pritee Khanna," Occlusion Invariant Palmprint Recognition with ULBP Histograms", IMCIP, 2015.

[22] Badrinath G. S.," Palmprint based Verification System Robust to Occlusion using Low-order Zernike Moments of Sub-images", BMVC 2009.

[23] Deepti Tamrakar and Pritee Khanna," Palmprint verification using competitive index with PCA", ICSCCN, September 2011.

[24] Deepti Tamrakar and Pritee Khanna," Noise and rotation invariant RDF descriptor for palmprint identification”, Springer, March 2015. 